\title{
Non-linear composition and infinite conformal symmetry of topologically non-trivial solutions in $(3+1)$-dimensional Yang-Mills theory
}

\author{
Fabrizio Canfora ${ }^{\mathrm{a}}$ \\ Centro de Estudios Científicos (CECS), Casilla 1469, Valdivia, Chile
}

Received: 6 October 2021 / Accepted: 15 November 2021 / Published online: 25 November 2021

(C) The Author(s) 2021

\begin{abstract}
An infinite-dimensional family of analytic solutions in pure $S U$ (2) Yang-Mills theory at finite density in $(3+1)$ dimensions is constructed. It is labelled by two integeres ( $p$ and $q$ ) as well as by a two-dimensional free massless scalar field. The gauge field depends on all the 4 coordinates (to keep alive the topological charge) but in such a way to reduce the (3+1)-dimensional Yang-Mills field equations to the field equation of a 2D free massless scalar field. For each $p$ and $q$, both the on-shell action and the energy-density reduce to the action and Hamiltonian of the corresponding 2D CFT. The topological charge density associated to the nonAbelian Chern-Simons current is non-zero. It is possible to define a non-linear composition within this family as if these configurations were "Lego blocks". The non-linear effects of Yang-Mills theory manifest themselves since the topological charge density of the composition of two solutions is not the sum of the charge densities of the components. This leads to an upper bound on the amplitudes in order for the topological charge density to be well-defined. This suggests that if the temperature and/or the energy is/are high enough, the topological density of these configurations is not well-defined anymore. Semiclassically, one can show that (depending on whether the topological charge is even or odd) some of the operators appearing in the 2D CFT should be quantized as Fermions (despite the Bosonic nature of the classical field).
\end{abstract}

\section{Contents}

1 Introduction . . . . . . . . . . . . . . 1

2 Action and ansatz . . . . . . . . . . 2

2.1 Spherically symmetric example . . . . . . . . 2

2.2 Non-spherical hedgehog approach . . . . . . . 3

3 Topological density and charge ......... 4

a e-mail: canfora@cecs.cl (corresponding author)
4 Energy-momentum tensor and topological density . 5

5 Allowed values of the topological charge . . . . . 6

6 Semi-classical considerations . . . . . . . . . 6

7 Conclusions . . . . . . . . . . . 8

References . . . . . . . . . . . . . . . . 8

\section{Introduction}

A proper analytic understanding of formation and interactions of topologically non-trivial configurations in nonAbelian gauge theories (especially at finite density) is one of the main open problems in high energy physics with implications both for the phases diagram of gauge theories and for the color confinement mechanism. Color confinement is closely related to topologically non-trivial configurations (see [1-9] and references therein) while, due to asymptotic freedom, at high energies Quark and Gluon should be liberated and Hadrons are melted [10-12]. It has been possible to create deconfined hadronic matter at high temperature in relativistic heavy-ion colliders (see for instance [13-16] and [17]) but also there non-perturbative effects (see [18] and references therein) dominate. The lack of analytic control on such effects in gauge theories is (partially) compensated by the great advances in lattice QCD (LQCD) (see [19-28] and references therein).

However, there are fundamental open issues (such as formation and dynamics of configurations with topological charge at finite density/volume and their temperature dependence) where analytic results would shed considerable new light on the phase diagram of QCD (as in these dynamical situations even lattice simulations are extremely challenging). For instance, numerical lattice simulations show that the leading corrections to an "ideal" gas term $T^{4}$ in four dimensions are terms quadratic in the temperature $\approx T^{2}$. This feature (which is certainly of non-perturbative origin) 
is not well understood yet: see [29-33]. Other relevant questions are: what happens when two (or more) topologically non-trivial configurations collide? What happens to the topological charge? and so on. From the analytic viewpoint, the situation could appear to be hopeless. In (3+1)-dimensional Yang-Mills theory, already the collision of (topologically trivial) plane waves is intractable and numerical methods must be used ${ }^{1}$ (see [34-40] and references therein). Thus, one might think that the analysis of processes (such as collisions) of solitonic-like configurations with non-vanishing topological charge should be "even more intractable" with analytic methods. ${ }^{2}$

In fact, in the present paper an infinite family of analytic and topologically non-trivial solutions of Yang-Mills theory with quite remarkable properties will be constructed. Such configurations possess an infinite conformal symmetry, allow a non-linear composition law (giving rise to a closed description of head-on collisions between these configurations) and also (through such composition law) allow to compute the critical amplitude beyond which the topological charge density become ill-defined.

The departure from spherical symmetry is a necessary ingredient. A systematic tool to construct non-spherical hedgehog ansatz are suitable to describe finite density effects have been developed in [43-53] for the Skyrme model [5456] (which is the low energy limit of QCD [57-63] at leading order in the 't Hooft expansion). Such strategy is quite effective in the Einstein-Yang-Mills case as well [64-66]. Here this approach will be adapted to the situation in which the $(3+1)$-dimensional Yang-Mills theory is defined within a flat region of finite spatial volume.

The paper is organized as follows: in the second section, the action and the ansatz will be described. In the third section the topological charge and density will be analyzed. In the fourth section the properties of the energy-momentum tensor will be discussed. In the fifth section, the allowed values of the topological charge will be studied. In the sixth section, some semi-classical considerations will be included. In the seventh section, some conclusions will be presented.

\footnotetext{
1 In the collision of wave packets it is known since the early eighties that for small amplitude wave packets, these pass through each other without being destroyed, although it is impossible to compute analytically in classical Yang-Mills theory in $(3+1)$ dimensions (the quantum problem being of course more difficult) the critical amplitude after which new kinds of configurations arise.

2 Indeed, these issues can only be dealt numerically already in the analysis of head-on collisions of (1+1)-dimensional kinks (which is far simpler than Yang-Mills theory in $(3+1)$-dimensions): see $[41,42]$ and references therein.
}

\section{Action and ansatz}

The Yang-Mills theory is defined by the action (here we will consider the $S U(2)$ case but the present results can be extended to the $S U(N)$ case)

$I=\frac{1}{2 e^{2}} \int d^{4} x \sqrt{-g} \operatorname{Tr}\left(F_{\mu \nu} F^{\mu \nu}\right)$,

where

$$
\begin{aligned}
F_{\mu \nu} & =\partial_{\mu} A_{\nu}-\partial_{\nu} A_{\mu}+\left[A_{\mu}, A_{\nu}\right], \\
A & =A_{\mu} d x^{\mu}=A_{\mu}^{j} t_{j} d x^{\mu}, \mathbf{t}_{j}=i \sigma_{j} .
\end{aligned}
$$

Here $e$ is the Yang-Mills coupling constant and the matrices $\mathbf{t}_{j}$ are the generators of the $S U$ (2) group being $\sigma_{j}$ the Pauli matrices. The field equations read

$\nabla_{\nu} F^{\mu \nu}+\left[A_{\nu}, F^{\mu \nu}\right]=0$.

The energy-momentum tensor is given by

$T_{\mu \nu}=-\frac{2}{e^{2}} \operatorname{Tr}\left(F_{\mu \alpha} F_{\nu}{ }^{\alpha}-\frac{1}{4} g_{\mu \nu} F_{\alpha \beta} F^{\alpha \beta}\right)$.

\subsection{Spherically symmetric example}

Here the usual spherically symmetric ansatz on flat spaces for the gauge field will be described (this subsection is useful to compare and contrast the usual case with the non-spherical ansatz needed at finite volume). Let us consider a flat spacetime described by the metric

$d s^{2}=-d t^{2}+d R^{2}+R^{2}\left(d \Theta^{2}+\sin ^{2} \Theta d \Phi^{2}\right)$,

where $R$ is the usual radial coordinate while $\Theta$ and $\Phi$ are the usual polar angular coordinates of flat space-times (only in this subsection, $R$ together with capital greek letters are used in order to avoid confusion with the coordinates system which will be used at finite density in the next sections). The most convenient ansatz for the non-Abelian gauge potential adapted to spherical symmetry is the following (see [6] for a pedagogical introduction):

$A_{\mu}=\lambda(R) U^{-1} \partial_{\mu} U$.

The function $\lambda(R)$ is called soliton profile: when $\lambda$ is either 0 or 1 the gauge field is trivial as it either vanishes or becomes a pure gauge, respectively. Consequently, $\lambda$ encodes (part of) the informations about the gauge field (especially, the informations about how the energy-density decays). On the other hand, the "pure gauge part" $U^{-1} \partial_{\mu} U$ also carries relevant informations in determining the non-Abelian fluxes. In particular, when $U(x) \in S U(2)$ reads

$$
\begin{aligned}
& U\left(x^{\mu}\right)=n^{i} t_{i}, \quad n^{i} n_{i}=1, \\
& n^{1}=\sin \Theta \cos \Phi, \quad n^{2}=\sin \Theta \sin \Phi, \quad n^{3}=\cos \Theta,
\end{aligned}
$$


two nice things happen. Firstly, the complete set of YangMills field equations reduce to just one equation for the profile $\lambda$. Secondly, the gauge field possesses a non-vanishing magnetic flux (determined by the two-form $\Omega=d \Theta \wedge d \Phi$ ). In other words, the magnetic flux is non-vanishing across the two-dimensional surfaces determined by the condition

$\Omega \neq 0$.

More in general, the gauge field will possess non-trivial fluxes in different directions when the " $U$ " used to build the "pure gauge" part of the ansatz (namely, the Maurer-Cartan form $U^{-1} \partial U$ ) possesses a non-trivial topological density $\rho_{Q}(U):$

$$
\begin{aligned}
\rho_{Q}(U) & =\left(U^{-1} \partial U\right)^{3} \\
& =\operatorname{Tr}\left\{\varepsilon_{i j k}\left(U^{-1} \partial_{i} U\right)\left(U^{-1} \partial_{j} U\right)\left(U^{-1} \partial_{k} U\right)\right\} .
\end{aligned}
$$

The integral of the above density over a space-like threedimensional hypersurface is the third homotopy class of $U$. In a sense, $\rho_{Q}(U)$ is a local measure of the genuine three-dimensional nature of the configuration since, when $\rho_{Q}(U) \neq 0$, the $S U(2)$-valued field can be used to build, locally, a three-dimensional volume form. A further comment about the spherical case is in order: the Maurer-Cartan form $U^{-1} \partial U$ can be expanded in terms of the right-invariant one-forms $\Omega_{\mu}^{j}$ along the basis of the algebra:

$U^{-1} \partial U=\sum_{j} \Omega_{\mu}^{j} \frac{\mathbf{t}_{j}}{2}$.

Thus, a natural basis to write down an ansatz for the gauge field is of course provided by the $\Omega_{\mu}^{j}$. In the spherically symmetric case, one can see that the three $\Omega_{\mu}^{j}$ "have the same weight" in the ansatz (in other words, one needs only one profile $\lambda(R)$ to make the field equations consistent). This is of course related with the well known spherical symmetry up to internal rotation of the ansatz. On the other hand, if one is interested in non-spherical situations (which are relevant, for instance, at finite density and, more in general, when non-trivial boundaries are present) one should expect that a consistent ansatz should include, at least, two different profiles (namely, different weight for the $\Omega_{\mu}^{j}$ ). This apparently obvious observation leads to the novel ansatz which is able to disclose an infinite-dimensional family of topologically non-trivial configuration in (3+1)-dimensional Yang-Mills theory at finite Baryon density.

\subsection{Non-spherical hedgehog approach}

The main goal of the paper is to construct a formalism able to describe how topologically non-trivial configurations of Yang-Mills theory react when they are forced to live within a finite box (an issue which must be addressed in the finite density analysis). The most natural way to take into account finite volume effects is to use the flat metric defined below:

$d s^{2}=-d t^{2}+A\left(d r^{2}+d \theta^{2}\right)+L^{2} d \phi^{2}$,

where $A^{1 / 2}$ and $L$ are constants with dimension of length (such that $8 \pi^{3} A L$ represents the volume of the box), while $r, \theta$ and $\phi$ are dimensionless coordinates with ranges

$0 \leq \theta \leq 2 \pi, \quad 0 \leq r \leq 4 \pi, \quad 0 \leq \phi \leq \pi$.

The above ranges for the coordinates $\theta, \phi$ and $r$ are related to the Euler angle parametrization for $S U(2)$ valued fields. Let us define the following $U \in S U(2)$ :

$U=\exp \left(p \theta \frac{\mathbf{t}_{3}}{2}\right) \exp \left(H(t, \phi) \frac{\mathbf{t}_{2}}{2}\right) \exp \left(q r \frac{\mathbf{t}_{3}}{2}\right)$.

where $p$ and $q$ are non-vanishing integers (there will be one more restriction on $p q$ which will be discussed later on). The theory of Euler angles for $S U(N)$ [67-69] tells that (when $p$ and $q$ are non-vanishing integers) the range of $\theta$ (appearing in the left factor of the decomposition in Eq. (10)) and the range of $r$ (appearing in the right factor of the decomposition in Eq. (10)) must be as in Eq. (9). As far as the central factor (namely $H(t, \phi))$ is concerned, there are two options. If the field $H(t, \phi)$ satisfies either periodic boundary conditions

$H(t, \phi=0)=H(t, \phi=\pi)$

or Dirichlet boundary conditions

$H(t, \phi=0)=H_{0}=H(t, \phi=\pi)$

with the same value $H_{0}$ at $\phi=0$ and $\phi=\pi$ then the topological charge of the configuration of the gauge field (which will be defined in the next section) vanishes (although the topological density can still be non-trivial). The other boundary condition for $H(t, \phi)$ arises naturally taking into account that $H(t, \phi)$ appears in the central factor of the Euler angles decomposition of an $S U(2)$ element (see [67-69]):

$H(t, \phi=0)=0, H(t, \phi=\pi)=\pi$,

or

$H(t, \phi=0)=\pi, H(t, \phi=\pi)=0$.

The two options here above ensure that the $S U(2)$ values element $U$ defined in Eqs. (9), (10) and (13) wraps an integer number of times around the group manifold of $S U(2)$ (in other words, $U$ has a non-vanishing winding number). In this case both the topological charge and the topological density associated to the gauge field (to be defined in the next section) will be non-trivial. 


\section{Topological density and charge}

Relevant topological properties of a given ansatz for a nonAbelian gauge field are encoded in Chern-Simons current:

$J_{\mu}^{C S}=\frac{1}{8 \pi^{2}} \varepsilon_{\mu \nu \rho \sigma} \operatorname{Tr}\left(A_{\nu} \partial_{\rho} A_{\sigma}+\frac{2}{3} A_{\nu} A_{\rho} A_{\sigma}\right), \rho_{B}=J_{0}^{C S}$,

where $\rho_{B}$ is the corresponding topological density and its integral along a space-like hypersurface is the topological charge.

On the one hand, the topological density of plane waves as well as colliding plane waves in Yang-Mills theory [34-40] is identically zero. On the other hand, it is well known (see [7073] and references therein) that $\rho_{B}$ is the "non-perturbatively induced Baryonic charge" of the gauge configuration [74]. Hence, it is clear that the above topological current (as well as the corresponding topological density $\rho_{B}$ ) encode very important properties of the gauge field.

For these reasons, in the present work the sentence "topologically non-trivial" refers to configurations with $\rho_{B} \neq$ 0 : configurations with vanishing total Baryonic charge but non-vanishing $\rho_{B}$ still describe complex patterns in which both regions with positive and negative charge densities are present and interact. Thus, even when the overall topological charge is vanishing, one can still learn a lot on the behavior of these non-perturbative configurations as long as $\rho_{B} \neq 0$.

Hence, we need an ansatz such that $J_{\mu}^{C S}$ is non-zero and, at the same time, the field equations can be solved analytically. Naively, one could think that the situation is hopeless since the two requirements (to have an ansatz with non-vanishing $\rho_{B}$ and, at the same time, leading to solvable Yang-Mills field equations) are, at a first glance, in conflict. The reason is that a non-zero $\rho_{B}$ requires at least three independent degrees of freedom in the ansatz depending on different spatial coordinates while we already know that even the collision of plane-waves in Yang-Mills theory necessarily involves numerical analysis. And yet, following [44,45,52,53,64-66] one arrives at the following ansatz for $A_{\mu}$ :

$A_{\mu}=\sum_{j=1}^{3} \lambda_{j} \Omega_{\mu}^{j} \mathbf{t}_{j}, U^{-1} \partial_{\mu} U=\sum_{j=1}^{3} \Omega_{\mu}^{j} \mathbf{t}_{j}$,

where

$$
\begin{aligned}
& H(t, \phi)=\arccos (G), G=G(t, \phi), \\
& \lambda_{1}(t, \phi)=\lambda_{2}(t, \phi) \\
& =\frac{G}{\sqrt{G^{2}+\exp 2 \eta}} \stackrel{\text { def }}{=} \lambda(t, \phi), \quad \lambda_{3}(t, \phi)=1, \eta \in \mathbb{R} \\
& G(t, \phi)=\exp 3 \eta \frac{F}{\sqrt{1-(\exp 4 \eta) \cdot F^{2}}}, F=F(t, \phi) .
\end{aligned}
$$

As far as the boundary conditions for $F(t, \phi)$ are concerned, the two options in Eqs. (11) and (12) give rise to the following two boundary conditions for $F(t, \phi)$ :

$F(t, \phi=0)=F(t, \phi=\pi)$

or Dirichlet boundary conditions

$F(t, \phi=0)=F_{0}=F(t, \phi=\pi)$

with the same $F_{0}$ at $\phi=0$ and $\phi=\pi$ : in this case the topological charge (which is the spatial integral of $\rho_{B}$ in Eq. (26) in the next section) vanishes. On the other hand, the option in Eq. (13), in terms of $F(t, \phi)$, reads:

$$
F(t, \phi=0)=-\frac{\exp (-2 \eta)}{\sqrt{1+\exp (2 \eta)}}, F(t, \phi=\pi)=\frac{\exp (-2 \eta)}{\sqrt{1+\exp (2 \eta)}} .
$$

In this case both the topological charge and the topological density will be non-trivial.

It is important to emphasize that these are not the only possible boundary conditions. For instance (especially in collision processes) one may wish to consider dynamical situations in which the topological charge inside the box we are considering is time-dependent (as there is an in-flux and/or out-flux of topological charge along the $\phi$-direction). The intuitive picture in these cases is of one (or more) topologically non-trivial configuration(s) moving in the $\phi$-direction in such a way that these configurations only spend a finite amount of time in the interval $0 \leq \phi \leq \pi$ (from the CFT viewpoint, these situations are better described in terms of Neumann or Robin boundary conditions for $F$ instead of Dirichlet). In these cases both $F(t, \phi=0)$ and $F(t, \phi=\pi)$ may depend on time (which would imply that the Baryon charge contained in the box defined in Eqs. (8) and (9) also depends on time: see Eq. (30) below). I will come back on these more general boundary conditions in a future publication.

The components of the gauge field can be easily computed taking into account the well known expression of the $\Omega_{\mu}^{j}$ in the case of the Euler parametrization. Thus, explicitly, $A_{\mu}$ reads

$$
\begin{aligned}
A_{\mu}= & \lambda(t, \phi)\left[\frac{\mathbf{t}_{1}}{2}(-\sin (q r) d H+p \cos (q r) \sin (H) d \theta)\right. \\
& \left.+\frac{\mathbf{t}_{2}}{2}(\cos (q r) d H+p \sin (q r) \sin (H) d \theta)\right] \\
& +\frac{\mathbf{t}_{3}}{2}(q d r+p \cos H d \theta), \\
d H= & \frac{\partial H}{\partial t} d t+\frac{\partial H}{\partial \phi} d \phi .
\end{aligned}
$$

The fact that $d \lambda \wedge d H=0$ together with the fact that the gradients of the coordinates $r, \theta$ and $\phi$ are mutually orthogonal simplifies many of the computations. The above ansatz 
is the key to get the results of the main paper. The rest is a direct computation.

With the above ansatz for the gauge field, the complete set of (3+1)-dimensional Yang-Mills field equations with the ansatz in Eqs. (10), (15), (16), (17) and (18) reduces to the field equation of a free massless scalar field in two dimensions:

$$
\square F=\left(\frac{\partial^{2}}{\partial t^{2}}-\frac{1}{L^{2}} \frac{\partial^{2}}{\partial \phi^{2}}\right) F=0 .
$$

\section{Energy-momentum tensor and topological density}

The surprises related to the ansatz in Eqs. (10), (15), (16), (17) and (18) do not finish in Eq. (23). Indeed, at a first glance, one could naively think that the resulting Yang-Mills equations are so simple because the configurations are topologically trivial. In fact, the topological density is non-vanishing (as long as $\frac{\partial F}{\partial \phi} \neq 0$ : see Eq. (26) here below). Explicitly, the energy density $T_{00}$ and the on-shell Lagrangian $L_{\text {on-shell }}$ reduce to the Hamiltonian and the Lagrangian of the corresponding CFT:

$$
\begin{aligned}
T_{00} & =\frac{p^{2}}{e^{2} A}(\exp 5 \eta) \cosh (\eta)\left[\left(\frac{\partial F}{\partial t}\right)^{2}+\frac{1}{L^{2}}\left(\frac{\partial F}{\partial \phi}\right)^{2}\right], \\
L_{\text {on-shell }} & =\frac{p^{2}}{e^{2} A}(\exp 5 \eta) \cosh (\eta)\left[\left(\frac{\partial F}{\partial t}\right)^{2}-\frac{1}{L^{2}}\left(\frac{\partial F}{\partial \phi}\right)^{2}\right] .
\end{aligned}
$$

A direct computation reveals that the topological density is

$$
\rho_{B}=\frac{p q(\exp 3 \eta)}{16 \pi^{2}\left[1-(\exp 4 \eta) F^{2}\right]^{3 / 2}} \frac{\partial F}{\partial \phi} .
$$

Note that, in the Abelian case the case, the Chern-Simons current would only have terms linear and quadratic in the gauge potential: thus the non-linear character of $\rho_{B}$ as function of $F$ in Eq. (26) is a genuine non-Abelian feature of the present family of configurations. It is also worth emphasizing that, in the topological density, both the term " $A F$ " and the term " $A A A$ " in the definition of Chern-Simons current in Eq. (14) are non-vanishing. In particular, the second term " $A A A$ " has strong similarities with the topological charge in Skyrme theory (see $[54-56,58,59])$. Indeed, the $U^{-1} \partial U$ part of the present ansatz has been constructed using as inspiration the topologically non-trivial solutions of the Skyrme model found in [43,45,49-53].

The full energy-momentum tensor reads:

$$
T_{\mu \nu}=\left[\begin{array}{cccc}
T_{00} & 0 & 0 & P_{\phi} \\
0 & T_{r r} & 0 & 0 \\
0 & 0 & T_{\theta \theta} & 0 \\
P_{\phi} & 0 & 0 & T_{\phi \phi}
\end{array}\right],
$$

where

$$
\begin{aligned}
T_{r r} & =\frac{p^{2}}{e^{2}}(\exp 5 \eta) \cosh (\eta)\left[\left(\frac{\partial F}{\partial t}\right)^{2}-\frac{1}{L^{2}}\left(\frac{\partial F}{\partial \phi}\right)^{2}\right] \\
& =-T_{\theta \theta}, \\
T_{\phi \phi} & =\frac{p^{2}}{e^{2} A}(\exp 5 \eta) \cosh (\eta)\left[L^{2}\left(\frac{\partial F}{\partial t}\right)^{2}+\left(\frac{\partial F}{\partial \phi}\right)^{2}\right] \\
T_{t \phi} & =P_{\phi}=\frac{p^{2}}{A e^{2}}(\exp 4 \eta)(1+\exp (2 \eta)) \frac{\partial F}{\partial t} \frac{\partial F}{\partial \phi}
\end{aligned}
$$

Hence, one can easily see that the energy-momentum tensor is traceless: $g^{\mu \nu} T_{\mu \nu}=0$ as it should in Yang-Mills theory in $(3+1)$-dimensions. It is also interesting to note that if one "eliminates" the coordinates $r$ and $\theta$, the resulting twodimensional energy-momentum tensor in the $t$ and $\phi$ direction is still traceless (as it happens for a two-dimensional conformal field theory). In other words, one can take $T_{a b}$ defined as

$T_{a b}=\left(\begin{array}{cc}T_{00} & P_{\phi} \\ P_{\phi} & T_{\phi \phi}\end{array}\right), \quad a, b=t, \phi$

as the effective energy-momentum tensor associated to the massless two-dimensional scalar field $F$.

From the expression in Eq. (29) it is clear that a solution of the field equations of the form $F_{1}=F_{1}(t / L-\phi)$ has opposite momentum with respect to a solution of the field equations of the form $F_{2}=F_{2}(t / L+\phi)$. Consequently, using such solutions $F_{1}$ and $F_{2}$ of Eq. (23) (which give rise to two gauge fields $A_{1}$ and $A_{2}$ through (10), (15), (16), (17) and (18)) one can define the composition of $F_{1}$ and $F_{2}$ replacing in Eqs. (10), (15), (16), (17) and (18) $F_{1}+F_{2}$ (or, in fact, any linear combination of $F_{1}$ and $F_{2}$ ) in place of $F$. The solution corresponding to $F_{1}+F_{2}$ describes the head-on collision of the two original solutions with opposite momentum.

From Eq. (26) it follows that the topological charge density associated to $F_{1}+F_{2}$ is the sum of the topological charge density associated to $F_{1}$ plus to one associated to $F_{2}$ only for small amplitudes (namely, $\left.\left|(\exp 4 \eta) F(t, \phi)^{2}\right| \ll 1\right)$.

Since the topological charge density in itself (and not only the topological charge) has the physical meaning of the (anomalous contribution to the) Baryonic charge density, it makes sense to ask what happens when the amplitude is not small. In this case, non-linear effects cannot be neglected. For instance, as it has been emphasized in the main text, the sum of $F_{1}(\phi-t / L)$ and $F_{2}(\phi+t / L)$ (such that both $F_{1}$ and $F_{2}$ possess a well-defined $\rho_{B}$ ) represents the head-on collision of the two gauge potentials associated to $F_{1}(\phi-t / L)$ and $F_{2}(\phi+t / L)$ (as these two configurations have opposite momenta). Using the fact the $F$ satisfies a linear equation, one can construct examples where the $\rho_{B}$ associated to $F_{1}+F_{2}$ becomes ill-defined. Configurations where $\left|(\exp 4 \eta)\left(F_{1}(t, \phi)+F_{2}(t, \phi)\right)^{2}\right|>1$ somewhere 
in the bulk (but such that $\left|(\exp 4 \eta) F_{j}(t, \phi)^{2}\right|<1$ everywhere) discloses inelastic behavior in a very similar way as the numerical results found in [40] (where the authors studied the head-on collisions of wave-packets).

A natural question here arises: what is the physical relevance of head-on collisions of topological objects? The interest of this issue arises from the fact that head-on scattering processes in non-integrable theories (such as Yang-Mills theory in (3+1)-dimensions) are extremely complicated both at classical and quantum level (so much so that even in much simpler models in $(1+1)$-dimensions only numerical methods have been able, until recently, to detect the arising of non-elastic thresholds). Classically, from the intuitive viewpoint, one can think at "non-elastic thresholds" as situations in which the scattering of classical objects (such as kinks) produces, in the final state, other objects as well (besides the original kinks). For instance, when two kinks collide (if their amplitude is large enough), in the final state one can have two kinks plus radiation. Unfortunately, to find analytically how large the amplitude of the kinks should be in order to see "new degrees of freedom" is a very difficult problem. It is therefore very interesting that, in this framework, it is possible to find explicitly the amplitude of the classical colliding solutions beyond which new degrees of freedom must appear: in the case of the present manuscript, such critical amplitude can be easily identified as the amplitude beyond which the (3+1)-dimensional topological charge density is not well defined anymore. This is the first analytic example of this kind.

\section{Allowed values of the topological charge}

The topological charge $B$ (which is the spatial integral of $\rho_{B}$ ) reads

$$
B=\left.\frac{(\exp 3 \eta)}{2} p q\left(\frac{F}{\sqrt{1-(\exp 4 \eta) \cdot F^{2}}}\right)\right|_{F(t, 0)} ^{F(t, \pi)} .
$$

As it has been already discussed, when $F(t, 0)=F(t, \pi)$ the topological charge vanishes. Thus, let us consider the boundary conditions for $F(t, \phi)$ in Eq. (21). The requirement to have an integer topological charge can be expressed as follows. The following auxiliary function is useful:

$$
\begin{aligned}
& \Omega(\eta, a, b) \stackrel{\text { def }}{=} \frac{\exp 3 \eta}{2}\left[\frac{a}{\sqrt{1-(\exp 4 \eta) \cdot a^{2}}}\right. \\
& \left.-\frac{b}{\sqrt{1-(\exp 4 \eta) \cdot b^{2}}}\right] .
\end{aligned}
$$

The topological charge reads

$B=p q \cdot \Omega(\eta, a=F(t, \pi), b=F(t, 0))$.
Taking into account the boundary conditions for $F(t, \phi)$ in Eq. (21) (which arises from the theory of Euler angles) $\Omega(\eta, a=F(t, \pi), b=F(t, 0))$ can be further simplified, so that one arrives at the following expression for the topological charge:

$B=p q$.

Further constraints arise if one requires that not only the Baryonic charge but also the Baryonic density in Eq. (26) must be everywhere well defined as it will be discussed in the next section.

\section{Semi-classical considerations}

Before going into the semiclassical considerations, let us remind the usual mode expansion of the solutions of Eq. (23):

$$
\begin{aligned}
F_{+}= & \phi_{0}^{+}+v_{+}\left(\frac{t}{L}+\phi\right)+\sum_{n \neq 0} a_{n}^{+} \sin \left[n\left(\frac{t}{L}+\phi\right)\right] \\
& +b_{n}^{+} \cos \left[n\left(\frac{t}{L}+\phi\right)\right], \\
F_{-}= & \phi_{0}^{-}+v_{-}\left(\frac{t}{L}-\phi\right)+\sum_{n \neq 0} a_{n}^{-} \sin \left[n\left(\frac{t}{L}-\phi\right)\right] \\
& +b_{n}^{-} \cos \left[n\left(\frac{t}{L}-\phi\right)\right],
\end{aligned}
$$

where, as usual, $F_{+}$refers to the left movers and $F_{-}$to the right movers $\left(v_{ \pm}\right.$and $\phi_{0}^{ \pm}$being integration constants which must satisfy three constraints which will be discussed below). Hence, the most general topologically non-trivial configuration of the present sector arises replacing $F=F_{+}+F_{-}$here above into Eqs. (15), (10), (16), (17) and (18). In order to have a clear physical picture, it is convenient to choose ${ }^{3} a_{n}^{ \pm}$ and $b_{n}^{ \pm}$in such a way that

$\widetilde{F}(t, \phi=0)=\widetilde{F}(t, \phi=\pi)=0$,

where $\widetilde{F}(t, \phi)$ is the part of $F=F_{+}+F_{-}$coming from the sum over the integers $n$ in Eqs. (33) and (34). Therefore $B$ in Eq. (30) is non-zero when

$v_{+}-v_{-} \neq 0$.

In particular, $v_{ \pm}$and $\phi_{0}^{ \pm}$in Eqs. (33) and (34) must be chosen in such a way to satisfy Eq. (21):

$$
\begin{aligned}
& F(t, \phi=\pi)=\phi_{0}^{+}+\phi_{0}^{-}+\left(v_{+}+v_{-}\right) \\
& \frac{t}{L}=\frac{\exp (-2 \eta)}{\sqrt{1+\exp (2 \eta)}} \Rightarrow
\end{aligned}
$$

3 However, this is not the only choice at all. There are many other possible boundary conditions which are worth to be explored. I will come back on this issue in a future publication. 


$$
\begin{aligned}
& v_{+}+v_{-}=0, \phi_{0}^{+}+\phi_{0}^{-}=\frac{\exp (-2 \eta)}{\sqrt{1+\exp (2 \eta)}}, \\
& F(t, \phi=0)=\frac{\exp (-2 \eta)}{\sqrt{1+\exp (2 \eta)}}+\left(v_{+}-v_{-}\right) \pi \\
& =-\frac{\exp (-2 \eta)}{\sqrt{1+\exp (2 \eta)}} \Rightarrow \\
& v_{-}=\frac{\exp (-2 \eta)}{\pi \sqrt{1+\exp (2 \eta)}} .
\end{aligned}
$$

At classical level, this is the simplest possible choice of boundary conditions since it allows to clearly identify the terms which are responsible for the topological charge and which are not. However, there are plenty of different options which will be discussed in a forthcoming paper.

As usual, the semi-classical quantization [75-80] corresponds to the quantization of the on-shell action evaluated on the ansatz Eqs. (10), (15), (16), (17) and (18). Consequently, as it has been shown in the previous section, for any fixed value of the topological charge, the semiclassical quantization of the theory corresponds to the quantization of a free two dimensional scalar field. There are some intriguing differences however.

Firstly, in Eqs. (33) and (34) any term in the expansion corresponds to an exact solution of the $(3+1)$-dimensional Yang-Mills equations and not just to a solution of the linearized field equations. Therefore the Bosonic quantum operators $\alpha_{n}^{+},\left(\alpha_{m}^{+}\right)^{\dagger}$ and $\alpha_{n}^{-},\left(\alpha_{m}^{-}\right)^{\dagger}$ (which are annihilation and creation operators for the left and right movers, satisfying the obvious commutation relations, see [81]) are quantum operators which create exact solutions of the semiclassical Yang-Mills equations. This situation should be contrasted with the more common scenario in which, given a particular solution of the $(3+1)$-dimensional Yang-Mills equations, the small fluctuations (both at classical and quantum level) around the given classical configuration are solutions of the linearized field equations (while are not solutions of the exact field equations, unless of course the theory is just a free theory).

Secondly, the constant terms $\phi_{0}^{ \pm}$as well as the linear terms in $t$ and $\phi$ play an important role. To see this, let us remind that the Baryonic charge is $p q$ (where $p$ and $q$ are integers) however, the field equation for $F$ does depend neither on $p$ nor on $q$. Since $p$ and $q$ label different topological sectors, there will be annihilation and creation operators for the left and right movers (satisfying the obvious commutation relations) in each sector $p q$. Consequently, one should introduce an extra index $b=p q$ to identify the topological sector to which the operators belong (it is also natural to assume that operators belonging to different topological sectors commute):

$\alpha_{n}^{ \pm} \rightarrow \alpha_{n, b}^{ \pm},\left(\alpha_{n}^{ \pm}\right) \dagger \rightarrow\left(\alpha_{n, b}^{ \pm}\right) \dagger$

$$
b \neq b^{\prime} \Rightarrow\left[\alpha_{n, b}^{ \pm},\left(\alpha_{n^{\prime}, b^{\prime}}^{ \pm}\right) \dagger\right]=0=\left[\alpha_{n, b}^{ \pm}, \alpha_{n^{\prime}, b^{\prime}}^{ \pm}\right] \forall n, n^{\prime}
$$

An interesting point here arises. According to [70-74], the topological charge $B=p q$ should be interpreted as Baryonic charge of the configuration. If this interpretation is accepted, then when $B$ is odd the configuration is a Fermion while when $B$ is even the configuration is a Boson. This observation has no consequences for the operators $\left(\alpha_{n, b}^{ \pm},\left(\alpha_{n^{\prime}, b^{\prime}}^{ \pm}\right) \dagger\right)$ since these operators are Bosonic in nature for any value of $B$ (as the corresponding classical solutions do not contribute to $B)$. On the other hand, the creation and annihilation operators associated to the linear part of the solution create a Boson or a Fermion depending on whether $B$ is even or odd. Hence, it is tempting to quantize $\phi_{0}^{ \pm}$and $v_{ \pm}$with commutators or anticommutators depending on $B$. In this sense, the quantization of $F$ would correspond to an "emergent superfield" when $B$ is odd since Fermionic $\left(\phi_{0}^{ \pm}\right.$and $\left.v_{ \pm}\right)$and Bosonic $\left(\alpha_{n, b}^{ \pm},\left(\alpha_{n^{\prime}, b^{\prime}}^{ \pm}\right) \dagger\right)$ operators would appear gathered on the same footing in $F$.

Let us consider for now the case in which $B$ is even so that all the operators are Bosonic. In this case, for any fixed $p$ and $q$, the semiclassical partition function (when the temperature is small enough) will coincide with the one of a massless scalar field in two dimensions. Obviously, the pressure and the grand partition function will be proportional to $T^{2}$. Consequently, the contribution of the present family of topologically non-trivial configuration to the grand partition function (as well as to the pressure) of Yang-Mills theory at finite volume is proportional to $T^{2}$.

The present framework also suggests that such a behavior cannot go on for very high temperatures and/or energies. One can see this using two intuitive arguments. However, before explaining these arguments, it is necessary to provide the classical condition to have a well defined Baryonic charge density

$(\exp 4 \eta) F^{2}(x) \leq 1$

(where, of course, $F(x)$ means $F(t, \phi)$ ) with a semiclassical interpretation. Hence, the classical field $F(x)$ (which is a solution of the field equation in Eq. (23)) is replaced by its quantization $\widehat{F}(x)$ (in which the coefficients of the expansions in Eqs. (33) and (34) are replaced by annihilation and creation operators for right and left movers, here the Baryonic charge is even so that all the relevant operators are Bosonic).

The first argument is related to the following semiclassical interpretation of the classical condition in Eq. (37) to have a well defined $\rho_{B}$ :

$$
(\exp 4 \eta) F^{2}(x) \leq 1 \rightarrow(\exp 4 \eta)\langle\widehat{F}(x) \widehat{F}(x+\varepsilon)\rangle \leq 1,
$$

where $\langle\widehat{F}(x) \widehat{F}(y)\rangle$ is the two-point function of the field quantum field $\widehat{F}$ and $\varepsilon$ is the UV cut-off. It is clear that the 
above condition will be violated for small enough $\varepsilon$. For the second argument one could interpret the classical condition in Eq. (37) as a bound on the one-point function of the composite operator $\widehat{F}^{2}$ at finite temperature:

$$
(\exp 4 \eta) F^{2}(x) \leq 1 \rightarrow(\exp 4 \eta)\left\langle\widehat{F}^{2}\right\rangle_{\beta} \leq 1,
$$

where $\langle\widehat{O}\rangle_{\beta}$ represents the vacuum expectation value (in the Euclidean theory) of the operator $\widehat{O}$ at temperature $1 / \beta$. Dimensional analysis tells that in a two-dimensional CFT the finite-temperature one point function of $\widehat{F}^{2}$ should behave as

$\left\langle\widehat{F}^{2}\right\rangle_{\beta} \approx(T L)^{2}$

where $L$ is the length in the $\phi$-direction in Eq. (8). Therefore, for not too large temperatures, the one-point function of $\widehat{F}^{2}$ is small for small temperatures. For large enough temperatures, $\left\langle\widehat{F}^{2}\right\rangle_{\beta}$ is large leading to the violation of Eq. (39). Thus, both in the UV and at high enough temperatures it could become problematic to define the topological charge densities.

\section{Conclusions}

In the present manuscript I have constructed an infinitedimensional family (labelled by two integers and a 2D free massless scalar field) of topologically non-trivial analytic solutions of (3+1)-dimensional Yang-Mills theory with pretty unique properties. The topological charge density (closely related to the Baryon density) associated to the nonAbelian Chern-Simons current in (3+1)-dimensions is nonzero and yet the complete set of (12, in the $S U$ (2) case) YangMills field equations reduce to the field equation of a $2 \mathrm{D}$ free massless scalar field. Moreover, these $(3+1)$-dimensional topologically non-trivial configurations can be manipulated as "Lego Blocks" as, given two solutions, one can get a new solution using a suitable non-linear composition law. Such non-linear composition law leads to a (semi-)classical upper bound on the amplitudes of the solutions related to the impossibility to define the topological charge density in the UV and/or at high enough temperatures. The semi-classical quantization of these configurations leads to the appearance of emergent Fermionic operators out of a purely Bosonic theory. These results open the surprising possibility to use the powerful analytic tools of 2D CFT to analyze $(3+1)$ dimensional Yang-Mills theory at finite Baryon density and temperatures in topologically non-trivial sectors.

Acknowledgements F. C. has been funded by Fondecyt Grant 1200022. The Centro de Estudios Cient íficos (CECs) is funded by the Chilean Government through the Centers of Excellence Base Financing Program of Conicyt.

Data Availability Statement This manuscript has no associated data or the data will not be deposited. [Authors' comment: There is no data will be deposited because this is mainly a theoretical work in which the main results are analytical in nature.]

Open Access This article is licensed under a Creative Commons Attribution 4.0 International License, which permits use, sharing, adaptation, distribution and reproduction in any medium or format, as long as you give appropriate credit to the original author(s) and the source, provide a link to the Creative Commons licence, and indicate if changes were made. The images or other third party material in this article are included in the article's Creative Commons licence, unless indicated otherwise in a credit line to the material. If material is not included in the article's Creative Commons licence and your intended use is not permitted by statutory regulation or exceeds the permitted use, you will need to obtain permission directly from the copyright holder. To view a copy of this licence, visit http://creativecomm ons.org/licenses/by/4.0/.

Funded by SCOAP ${ }^{3}$.

\section{References}

1. J. Greensite, An Introduction to the Confinement Problem, Lecture Notes in Physics, vol. 821. (Springer, Berlin, 2011)

2. N. Manton, P. Sutcliffe, Topological Solitons (Cambridge University Press, Cambridge, 2007)

3. A. Balachandran, G. Marmo, B. Skagerstam, A. Stern, Classical Topology and Quantum States (World Scientific, Singapore, 1991)

4. M. Shifman, Advanced Topics in Quantum Field Theory: A Lecture Course (Cambridge University Press, Cambridge, 2012)

5. M. Shifman, A. Yung, Supersymmetric Solitons (Cambridge University Press, Cambridge, 2009)

6. E.J. Weinberg, Classical Solutions in Quantum Field Theory (Cambridge, Cambridge, 2012)

7. H. Nielsen, P. Olesen, Nucl. Phys. B 144, 376 (1978)

8. G. t'Hooft, Nucl. Phys. B 79, 276 (1974)

9. A.M. Polyakov, JETP Lett. 20, 194 (1974)

10. N. Cabibbo, G. Parisi, Phys. Lett. B 59, 67 (1975)

11. D.J. Gross, F. Wilczek, Phys. Rev. Lett. 30, 1343 (1973)

12. H.D. Politzer, Phys. Rev. Lett. 30, 1346 (1973)

13. M. Gyulassy, in Structure and dynamics of elementary matter. Proceedings, NATO Advanced Study Institute, CamyuvaKemer, Turkey, September 22-October 2, 2003 (2004), p. 159-182. arXiv:nucl-th/0403032

14. E. Shuryak, Prog. Part. Nucl. Phys. 62, 48 (2009). arXiv:0807.3033 [hep-ph]

15. H. Satz, Lect. Notes Phys. 841, 1 (2012)

16. S.L. Shapiro, S.A. Teukolsky (1983) Black holes, white dwarfs, and neutron stars: the physics of compact objects

17. N.K. Glendenning, Compact stars: Nuclear physics, particle physics, and general relativity (1997)

18. N. Brambilla et al., Eur. Phys. J. C 74, 2981 (2014). arXiv:1404.3723 [hep-ph]

19. M.G. Alford, A. Kapustin, F. Wilczek, Phys. Rev. D 59, 054502 (1999). arXiv:hep-lat/9807039

20. J.B. Kogut, D.K. Sinclair, Phys. Rev. D 66, 014508 (2002). arXiv:hep-lat/0201017

21. J.B. Kogut, D.K. Sinclair, Phys. Rev. D 66, 034505 (2002). arXiv:hep-lat/0202028

22. J.B. Kogut, D.K. Sinclair, Phys. Rev. D 70, 094501 (2004). arXiv:hep-lat/0407027

23. S.R. Beane, W. Detmold, T.C. Luu, K. Orginos, M.J. Savage, A. Torok, Phys. Rev. Lett. 100, 082004 (2008). arXiv:0710.1827 [heplat]

24. W. Detmold, K. Orginos, Z. Shi, Phys. Rev. D 86, 054507 (2012). arXiv:1205.4224 [hep-lat] 
25. G. Endrodi, Phys. Rev. D 90, 094501 (2014). arXiv:1407.1216 [hep-lat]

26. M.J. Teper, Phys. Rev. D 59, 014512 (1999). arXiv:hep-lat/9804008

27. B. Lucini, M. Teper, Phys. Rev. D 66, 097502 (2002). arXiv:hep-lat/0206027

28. B. Bringoltz, M. Teper, Phys. Lett. B 645, 383 (2007). arXiv:hep-th/0611286

29. T. Umeda, S. Ejiri, S. Aoki, T. Hatsuda, K. Kanaya, Y. Maezawa, H. Ohno, Phys. Rev. D 79, 051501 (2009)

30. M. Panero, Phys. Rev. Lett. 103, 232001 (2009)

31. S. Borsanyi, G. Endrodi, Z. Fodor, S.D. Katz, K.K. Szabo, JHEP 1207, 056 (2012)

32. M. Caselle, L. Castagnini, A. Feo, F. Gliozzi, U. Gursoy, M. Panero, A. Schafer, JHEP 1205, 135 (2012)

33. D. Smith, A. Dumitru, R. Pisarski, L. von Smekal, Phys. Rev. D 88(5), 054020 (2013)

34. R.P. Treat, Nuovo Cimento A 6, 121-128 (1971)

35. Sidney R. Coleman, Phys. Lett. B 70, 59-60 (1977)

36. Y. Nutku, J. Phys. A Math. Gen. 16, L583-L586 (1983)

37. K. Rajagopal, N. Turok, Nucl. Phys. B 375, 299 (1992)

38. H. Goldberg, D. Nash, M.T. Vaughn, Phys. Rev. D 46, 2585 (1992)

39. C.R. Hu, S.G. Matinyan, B. Muller, A. Trayanov, T.M. Gould, S.D.H. Hsu, E.R. Poppitz, Phys. Rev. D 52, 2402 (1995)

40. C.R. Hu, S.G. Matinyan, B. Muller, D. Sweet, Phys. Rev. D 53, $3823(1996)$

41. P. Dorey, T. Romanczukiewicz, Phys. Lett. B 779, 117-123 (2018)

42. T.S. Mendonca, H.P. de Oliveira, JHEP 09, 120 (2015)

43. F. Canfora, Phys. Rev. D 88, 065028 (2013)

44. E. Ayon-Beato, F. Canfora, J. Zanelli, Phys. Lett. B 752, 201-205 (2016)

45. P.D. Alvarez, F. Canfora, N. Dimakis, A. Paliathanasis, Phys. Lett. B 773, 401-407 (2017)

46. L. Aviles, F. Canfora, N. Dimakis, D. Hidalgo, Phys. Rev. D 96, 125005 (2017)

47. F. Canfora, M. Lagos, S.H. Oh, J. Oliva, A. Vera, Phys. Rev. D 98(8), $085003(2018)$

48. F. Canfora, N. Dimakis, A. Paliathanasis, Eur. Phys. J. C 79(2), 139 (2019)

49. F. Canfora, Eur. Phys. J. C 78(11), 929 (2018)

50. F. Canfora, S.-H. Oh, A. Vera, Eur. Phys. J. C 79(6), 485 (2019)

51. F. Canfora, M. Lagos, A. Vera, Eur. Phys. J. C 80(8), 697 (2020)

52. P.D. Alvarez, S.L. Cacciatori, F. Canfora, B.L. Cerchiai, Phys. Rev. D 101(12), 125011 (2020)

53. E. Ayon-Beato, F. Canfora, M. Lagos, J. Oliva, A. Vera, Eur. Phys. J. C 80(5), 384 (2020)

54. T. Skyrme, Proc. R. Soc. Lond. A 260, 127 (1961)

55. T. Skyrme, Proc. R. Soc. Lond. A 262, 237 (1961)
56. T. Skyrme, Nucl. Phys. 31, 556 (1962)

57. C.G. Callan Jr., E. Witten, Nucl. Phys. B 239, 161-176 (1984)

58. E. Witten, Nucl. Phys. B 223, 422 (1983)

59. E. Witten, Nucl. Phys. B 223, 433 (1983)

60. A.P. Balachandran, V.P. Nair, N. Panchapakesan, S.G. Rajeev, Phys. Rev. D 28, 2830 (1983)

61. A.P. Balachandran, A. Barducci, F. Lizzi, V.G.J. Rodgers, A. Stern, Phys. Rev. Lett. 52, 887 (1984)

62. A.P. Balachandran, F. Lizzi, V.G.J. Rodgers, A. Stern, Nucl. Phys. B 256, 525-556 (1985)

63. G.S. Adkins, C.R. Nappi, E. Witten, Nucl. Phys. B 228, 552-566 (1983)

64. F. Canfora, S.-H. Oh, P. Salgado-Rebolledo, Phys. Rev. D 96(8), 084038 (2017)

65. F. Canfora, A. Gomberoff, S.-H. Oh, F. Rojas, P. SalgadoRebolledo, JHEP 06, 081 (2019)

66. F. Canfora, S.-H. Oh, Eur. Phys. J. C 81(5), 432 (2021)

67. S. Bertini, S.L. Cacciatori, B.L. Cerchiai, J. Math. Phys. (N.Y.) 47, 043510 (2006)

68. S.L. Cacciatori, F.D. Piazza, A. Scotti, Trans. Am. Math. Soc. 369, 4709 (2017)

69. T.E. Tilma, G. Sudarshan, J. Geom. Phys. 52, 263 (2004)

70. S.L. Adler, Phys. Rev. 177, 2426-2438 (1969)

71. J.S. Bell, R. Jackiw, Il Nuovo Cimento A 60(1), 47-61 (1969)

72. M.E. Shaposhnikov, G.R. Farrar, Phys. Rev. Lett. 70, 2833-2836 (1993)

73. V.A. Kuzmin, V.A. Rubakov, M.E. Shaposhnikov, Phys. Lett. B 155, 36-42 (1985)

74. F.R. Klinkhamer, N.S. Manton, Phys. Rev. D 30, 2212 (1984)

75. P.J. Ellis, J.I. Kapusta, H.-B. Tang, Phys. Lett. B 443, 63 (1998)

76. I.A. Shushpanov, J.I. Kapusta, P.J. Ellis, Phys. Rev. C 59, 2931 (1999)

77. I.T. Drummond, R.R. Horgan, P.V. Landshoff, A. Rebhan, Phys. Lett. B 460, 197 (1999)

78. N.O. Agasian, Phys. Lett. B 519, 71 (2001)

79. E. Megias, E. Ruiz Arriola, L.L. Salcedo, Phys. Rev. D 80, 056005 (2009)

80. V. Gogokhia, M. Vasuth, J. Phys. G Nucl. Part. Phys. 37, 075015 (2010)

81. M.B. Green, J.H. Schwarz, E. Witten, Superstring Theory (volume 1) (Cambridge University Press, Cambridge, 1988) 